\title{
Neuer Wirkstoff reduziert Entzündungsaktivität
}

— Für die Behandlung von Patienten mit chronisch obstruktiver Lungenerkrankung (COPD) könnte bald ein neues Medikament zur Verfügung stehen, das direkt an der zugrunde liegenden Entzündung ansetzt. Wie die Ergebnisse von vier kontrollierten Phase-III-Studien zeigen, reduziert die Therapie mit dem Phosphodiesterase-(PDE-)4Hemmer Roflumilast bei mittelschwerer bis schwerer COPD die Zahl der Exazerbationen signifikant und klinisch relevant und verbessert die Lungenfunktion.

Der PDE-4-Hemmer, dessen Zulassung mit dem Handelsnamen Daxas ${ }^{\circledR}$ in Europa beantragt ist, ist nach Ansicht von Prof. Claus Vogelmeier vom Universitätsklinikum Marburg ein interessantes pathophysiologisches Konzept. Denn er reduziert Entzündungsmediatoren, die beim Krankheitsgeschehen der COPD wahrscheinlich sehr wichtig sind.
In zwei der vier Zulassungsstudien wurden Patienten mit chronischem Husten und Auswurf über ein Jahr therapiert. Sie lagen mit ihrer Einsekundenkapazität $\left(\mathrm{FEV}_{1}\right)$ nach Gabe eines Bronchodilatators bei maximal 50\% des Sollwerts. Zudem hatte jeder im Jahr vor dem Studienstart mindestens eine moderate oder schwere Exazerbation.

In der gepoolten Analyse beider Untersuchungen senkte Roflumilast im Vergleich zu Placebo die Zahl der Exazerbationen, die mit Antibiotika oder systemischen Kortikosteroiden behandelt werden mussten, um $17 \%$. Zusätzlich besserte die einmal tägliche Einnahme des PDE-4-Hemmers die Lungenfunktion signifikant. Wurde die Tablette vor der Gabe eines Bronchodilatators eingenommen, besserte sich die $\mathrm{FEV}_{1}$ um im Schnitt $48 \mathrm{ml}$ im Vergleich zu Placebo. Bei der Einnahme nach der Anwendung des Bronchodilatators betrug der Anstieg
$55 \mathrm{ml}$. In zwei weiteren Studien über sechs Monate wurde Roflumilast versus Placebo zusätzlich zu den Standard-Bronchodilatatoren Salmeterol oder Tiotropium geprüft. Dabei ließ sich gegenüber Placebo eine Verbesserung des $\mathrm{FEV}_{1}$ um im Mittel $49 \mathrm{ml}$ bzw. $80 \mathrm{ml}$ beobachten. Ob sich durch eine systemisch wirkende Substanz wie den PDE-4-Hemmer auch die extrapulmonalen Komorbiditäten bei COPD beeinflussen lassen, muss laut Vogelmeier noch geprüft werden. Der PDE-4-Hemmer wurde gut vertragen. Die häufigsten Nebeneffekte waren Übelkeit, Diarrhö und Gewichtsverlust. „Dies trat aber nur bei wenigen und vor allem bei übergewichtigen Patienten auf", so Vogelmeier. bm

Update „Innovationen bei COPD und Asthma", 24.10.2009 in Bad Reichenhall, Veranstalter: Nycomed

Nicht kleinzelliges Bronchialkarzinom

\section{Die Histologie ermöglicht eine individualisierte Behandlung}

- Patienten mit nicht kleinzelligem Bronchialkarzinom (NSCLC) können heute aufgrund des histologischen Befundes besonders individuell behandelt werden. Zu den größten Fortschritten bei Patienten mit fortgeschrittenen Adeno- oder großzelligen Karzinomen zählt die Kombination des Multikinasehemmstoffs Pemetrexed mit Cisplatin in der Erstlinienbehandlung. Die Überlebenszeit der Patienten mit Adenokarzinomen konnte mit dieser Kombination in der Zulassungsstudie erstmals auf im Median über ein Jahr verlängert werden.

„Die Histologie ist zu einem wichtigen prädiktiven Faktor für die Selektion der Patienten für eine bestimmte Therapie geworden“, so Dr. Florian Länger, Pathologe an der MH Hannover. Denn das Ansprechen bei fortgeschrittenem NSCLC auf Pemetrexed (Alimta ${ }^{\circledR}$ ) variiert in Abhängigkeit der Histologie, wie Daten der Zulassungsstudie für die Ersttherapie in Kombination mit Cisplatin bei 1725 nicht zytostatisch vorbe- handelte Patienten belegen. Insgesamt war die Überlebenszeit jener, die mit Cisplatin $\left(75 \mathrm{mg} / \mathrm{m}^{2}\right)$ und Pemetrexed $\left(500 \mathrm{mg} / \mathrm{m}^{2}\right.$ jeweils am Tag 1) oder mit Cisplatin (Tag 1) und Gemcitabin (1250 mg/m² Tag 1 + 8) behandelt wurden (bis zu sechs Zyklen), nicht unterschiedlich, sagte Prof. Martin Wolf, Kassel. Bei Patienten mit Adenokarzinomen war im Pemetrexed-Arm die Überlebenszeit aber im Median um fast zwei Monate verlängert (12,6 vs. 10,9 Monate), bei großflächigen Karzinomen um fast vier (10,4 vs. 6,7 Monate). Bei Patienten mit Plattenepithel-Ca. war eine Pemetrexedtherapie von Nachteil (9,4 vs. 10,8 Monate), weshalb der Multikinasehemmer bei ihnen nicht indiziert ist [1]. Deutlich geringer war auch die Toxizität der Pemetrexedtherapie. Nur 12\% der Patienten dieser Gruppe hatten eine Alopezie im Vergleich zu 21\% im Gemcitabin-Cisplatin-Arm. Zudem gab es signifikant weniger Neutropenien, Hospit alisierungen, Transfusionen und Bedarf an

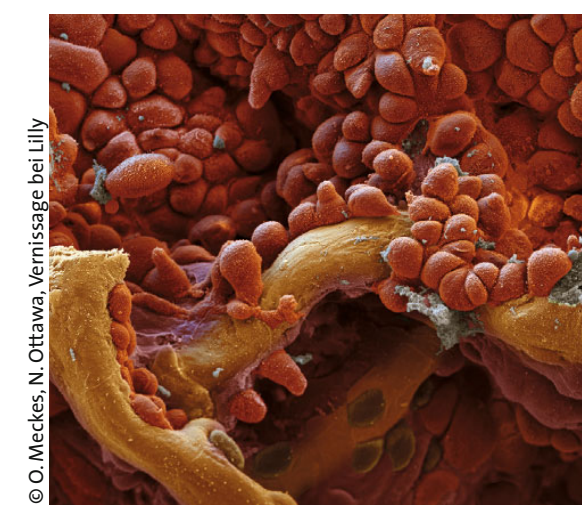

Pralle, mit kurzen Zilien besetzte Adenokarzinomzellen der Lunge (rot) in 1500facher Vergrößerung. Die glatten Zonen zeigen intaktes Alveolarepithel.

Wachstumsfaktoren. Außer zur Erstlinientherapie in Kombination mit Cisplatin ist Pemetrexed als Monotherapie auch für die Zweitlinientherapie bei fortgeschrittenem nicht Plattenepithel-Ca. zugelassen. $\quad$ rf

1. Scagliotti G et al. J Clin Oncol 2008;26:3543-51.

Pressegespräch „Histologie und individualisierte Therapie“ 26.11.2010 in Bad Homburg, Veranstalter: Lilly Deutschland 